\title{
INTERACTION OF A PLANE COMPRESSIONAL ELASTIC WAVE WITH A RIGID SPHEROIDAL INCLUSION*
}

\author{
BY \\ SUBHENDU K. DATTA \\ University of Colorado
}

\begin{abstract}
In this paper we have considered the problem of diffraction of a plane compressional harmonic elastic wave by a rigid spheroidal inclusion embedded in a homogeneous isotropic medium. For simplicity we have confined our attention to the axisymmetric case when the incident wave propagates along the axis of symmetry of the spheroid. The inclusion is assumed to be movable. Since the exact solution to this problem is not obtainable analytically we have used a boundary perturbation technique that is applicable at any finite frequency. We have derived exact analytical expression for the amplitude of oscillation of the inclusion correct to first order in a shape correction factor $\epsilon$. It is shown that the low-frequency expansion of the amplitude agrees with the expansion derived by other means correct to first order in frequency. We have also given a high-frequency expansion of the amplitude. Furthermore, we have derived the asymptotic expansion of the field in the illuminated zone and have shown that these are compatible with those obtained by an application of Keller's ray theory.
\end{abstract}

1. Introduction. The problem of diffraction of elastic waves in three dimensions by finite obstacles has been the subject of some recent studies. As is evidenced from the literature, most of these studies consider spherical inclusions [1-5]. The radiated waves from an oscillating rigid spherical inclusion have also received considerable attention in [6-9]. The departure from these are the studies concerned with the diffraction by rigid circular discs [10-12]. For the purposes of application, however, it is important to be able to analyze the diffraction by, and response of, an arbitrarily shaped inclusion. But these problems are not amenable to exact solutions, unless the vector elastodynamic equations are separable in the orthogonal coordinates associated with the shape of the boundary. Even then, unless the boundary is a sphere or a disc, the separation of variables technique leads to solutions in series of appropriate wave functions, which, on use of the boundary conditions, lead to an infinite set of linear algebraic equations for the determination of the unknown coefficients. This system of equations does not lend itself to the analytical determination of the unknown coefficients. So it seems that unless we have better mathematical tools available we have to use some suitable approximate methods.

One such method, applicable in very general situations, is the boundary perturbation technique which is used extensively in the electromagnetic diffraction problems (see [13]

* Peceived October 4, 1971; revised version received March 4, 1972. This research is supported by a grant from the National Science Foundation. 
for relevant references) and which has also recently been used by us [14, 15] in elastic problems. Its advantage is that its usefulness is not limited to low frequencies, although it cannot be applied at very high frequencies. At high frequencies, however, the ray theory of Keller or progressing wave expansions [16] can be used. For very low frequencies we also have various approximate methods available to us $[17,18]$.

The details of the method and other relevant references can be found in [14]. In the next section we apply this method to studying the response of an axisymmetric rigid inclusion to an incident plane harmonic compressional wave. There we present a formal solution of the problem in powers of a suitable shape correction factor $\epsilon$. In Sec. 3 we obtain the amplitude of oscillation $U$ of the inclusion in the particular case when it is a spheroid correct to first order in $\epsilon$, and show that the low-frequency expansion of $U$ agrees to $O(\alpha \mathrm{C})$ with that obtained by a simple method outlined in [19]. This method, however, gives $\mathrm{U}$ only to $O(\alpha \mathrm{C})$. Here $\mathrm{C}$ is a characteristic dimension of the body and $\alpha$ is a measure of the reciprocal of the wavelength of the disturbance. In Sec. 3 we also give a high-frequency expansion of $\mathrm{U}$. This expression agrees with that obtained for a sphere as $\epsilon \rightarrow 0$. In Sec. 4 we obtain the leading term, at high frequencies, of the field in the illuminated region and show in Sec. 5 that this agrees to $O(\alpha \mathrm{C} \epsilon)$ with the expansion in powers of $\alpha \mathrm{C} \epsilon$ of the field obtained by the ray theory of Keller et al. [20].

2. Method of solution. The displacement equations of motion in the linear theory of isotropic homogeneous elastic solids can be written as

$$
(\lambda+2 \mu) \nabla \nabla \cdot \mathbf{u}-\mu \nabla \wedge \nabla \wedge \mathfrak{u}=\rho\left(\partial^{2} / \partial t^{2}\right) \mathbf{u}
$$

where $\mathbf{u}(\mathbf{r}, t)$ is the displacement at position $\mathbf{r}, \lambda, \mu$ are Lamé constants, and $\rho$ is the density. We shall assume that $\mathbf{u}(\mathbf{r}, t)=\mathbf{u}(\mathbf{r}) \exp (-i \omega t)$, so that $\mathbf{u}(\mathbf{r})$ satisfies the equation

$$
(\lambda+2 \mu) \nabla \nabla \cdot \mathbf{u}-\mu \nabla \wedge \nabla \wedge \mathbf{u}+\rho \omega^{2} \mathbf{u}=0 .
$$

Suppose that the equation of the surface $S$ of the rigid inclusion $B$ is given in spherical polar coordinates $(r, \theta, \Phi)$ as

$$
r=\mathrm{C}(1+\epsilon f(\theta))
$$

where $\mathrm{C}, \epsilon$ are constants $(|\epsilon|<1), f(\theta)$ is continuous single-valued with continuous derivatives in $0 \leq \theta \leq \pi$ and satisfies the condition $|\epsilon f(\theta)|<1$. In writing (3) we have assumed that the origin of the coordinates system is on the axis of symmetry $(\theta=0, \pi)$ of $B$. It may be pointed out that any smooth surface $S$ which admits of a "radial singlevalued explicit representation" can be written in the form (3) by a suitable choice of C and a suitable location of the center of the "unperturbed sphere" $(r=\mathrm{C})$. This choice is obviously not unique.

In what follows we shall consider the response of the inclusion $\mathrm{B}$ to an incident compressional wave represented by

$$
\mathbf{u}^{(i)}(\mathbf{r})=\nabla \phi^{(i)}=-\frac{i u_{0}}{\alpha} \nabla \exp (i \alpha r \cos \theta), \quad \alpha=\omega\left(\frac{\rho}{\lambda+2 \mu}\right)^{1 / 2} .
$$

From symmetry it is seen that $\mathrm{B}$ will undergo rigid body oscillations along its axis. Let $\mathrm{U}=\mathrm{U} \exp (-i \omega t) \mathbf{i}_{z}$ be the displacement of $\mathrm{B}$ along its axis, $\mathrm{U}$ being the amplitude of oscillations (assumed to be small). 
Let $\mathfrak{u}^{(s)}(\mathbf{r})=\mathbf{v}(\mathbf{r})$ represent the time-independent part of the scattered field. Then on $S, \nabla(r)$ must satisfy the condition

$$
\mathbf{v}+\mathbf{u}^{(i)}=\mathbf{U}=\mathrm{U}(\hat{r} \cos \theta-\hat{\theta} \sin \theta),
$$

$\hat{r}, \hat{\theta}$ being unit vectors along the $r$ and $\theta$ directions, respectively. Furthermore, $\mathbf{v}(\mathbf{r})$ will satisfy a suitable radiation condition at infinity [21].

The solution of Eq. (2) that is independent of $\Phi$ and that satisfies the radiation condition can be written as

$$
\begin{gathered}
\nabla(\mathbf{r})=\nabla \chi+\nabla \wedge(-(\partial \psi / \partial \theta) \hat{\phi}), \\
\chi=\sum_{n=0}^{\infty} a_{n} h_{n}(\alpha r) P_{n}(\eta), \quad \psi=\sum_{n=0}^{\infty} b_{n} h_{n}(\beta r) P_{n}(\eta) .
\end{gathered}
$$

Here $\beta=\omega(\rho / \mu)^{1 / 2}, \eta=\cos \theta$, and $h_{n}(z)=(\pi / 2 z)^{1 / 2} H_{n+1 / 2}^{(1)}(z)$ is the spherical Bessel function of the third kind, $P_{n}(\eta)$ is the Legendre polynomial of degree $n$, and $P_{n}^{1}(\eta)$ is the associated Legendre function of the first kind of degree $n$ and order 1.

In the following we shall formally derive expressions for $a_{n}$ and $b_{n}$ in series of powers of $\epsilon$ so that $v$ given by (6) will satisfy (5). In order to do this let us assume*

$$
a_{n}=\sum_{p=0}^{\infty} \epsilon^{p} a_{n}^{p}, \quad b_{n}=\sum_{p=0}^{\infty} \epsilon^{p} b_{n}^{p} .
$$

Also, we shall expand $h_{n}(\alpha \mathrm{C}(1+\epsilon f))$, etc., in Taylor's series about $\alpha \mathrm{C}$ and write

$$
\begin{aligned}
h_{n}(\alpha \mathrm{C}(1+\epsilon f)) & =\sum_{p=0}^{\infty} \epsilon^{p} \frac{\mathrm{C}^{p} f^{p}}{p !} \alpha_{n}^{p}, \quad \alpha_{n}^{p}=\left[\frac{d^{p}}{d r^{p}} h_{n}(\alpha r)\right]_{r-\mathrm{c}}, \\
h_{n}(\beta \mathrm{C}(1+\epsilon f)) & =\sum_{p=0}^{\infty} \epsilon^{p} \frac{\mathrm{C}^{p} f^{p}}{p !} \beta_{n}^{p}, \\
\frac{1}{\mathrm{C}(1+\epsilon f)} h_{n}(\alpha \mathrm{C}(1+\epsilon f)) & =\sum_{p=0}^{\infty} \epsilon^{p} \frac{\mathrm{C}^{p} f^{p}}{p !} \gamma_{n}^{p}, \quad \gamma_{n}^{p}=\left[\frac{d^{p}}{d r^{p}}\left(\frac{h_{n}(\alpha r)}{r}\right)\right]_{r-\mathrm{o}}, \\
\frac{1}{\mathrm{C}(1+\epsilon f)} h_{n}(\beta \mathrm{C}(1+\epsilon f)) & =\sum_{p=0}^{\infty} \epsilon^{p} \frac{\mathrm{C}^{p} f^{p}}{p !} \delta_{n}^{p}, \\
j_{n}(\alpha \mathrm{C}(1+\epsilon f)) & =\sum_{p=0}^{\infty} \epsilon^{p} \frac{\mathrm{C}^{p} f^{p}}{p !} \mu_{n}^{p}, \\
\frac{1}{\mathrm{C}(1+\epsilon f)} j_{n}(\alpha \mathrm{C}(1+\epsilon f)) & =\sum_{p=0}^{\infty} \epsilon^{p} \frac{\mathrm{C}^{p} f^{p}}{p !} \nu_{n}^{p} .
\end{aligned}
$$

$\beta_{n}^{D}, \delta_{n}^{D}$ are obtained from $\alpha_{n}^{D}, \gamma_{n}^{D}$, respectively, on replacing $\alpha$ by $\beta . \mu_{n}^{D}, \nu_{n}^{D}$ are obtained from $\alpha_{n}^{p}, \gamma_{n}^{p}$, respectively, on replacing $h_{n}$ by $j_{n}$. Here $j_{n}(\alpha r)=(\pi / 2 \alpha r)^{1 / 2} J_{n+1 / 2}(\alpha r)$.

Using (9) and (8) in (6) and (7) we obtain, because of (5),

$$
\begin{aligned}
& \sum_{p=0}^{\infty} \epsilon^{p} \sum_{n=0}^{\infty} P_{n}(\eta) \sum_{a=0}^{p} \frac{\mathrm{C}^{a} f^{a}}{q !} \\
& \cdot\left[\alpha_{n}^{a+1} a_{n}^{p-a}+n(n+1) \delta_{n}^{a} b_{n}^{p-a}-\frac{i u_{0}}{\alpha} \imath^{n}(2 n+1) \mu_{n}^{a+1} \delta_{p a}\right]=\mathrm{U} P_{1}(\eta),
\end{aligned}
$$

* The superscript on a letter without subscript will denote the power to which that is to be raised. Otherwise, this will denote an index. 


$$
\begin{aligned}
\sum_{p=0}^{\infty} \epsilon^{p} \sum_{n=0}^{\infty} P_{n}^{1}(\eta) \sum_{a=0}^{p} \frac{\mathrm{C}^{a} f^{a}}{q !} \\
\cdot\left[\gamma_{n}^{a} a_{n}^{p-a}+\left(\delta_{n}^{a}+\beta_{n}^{a+1}\right) b_{n}^{p-a}-\frac{i u_{0}}{\alpha} i^{n}(2 n+1) \nu_{n}^{a} \delta_{p q}\right]=\mathrm{U} P_{1}^{1}(\eta) \\
\delta_{p a}=1, \quad p=q \\
=0, \quad p \neq q .
\end{aligned}
$$

If we now equate the coefficients of $\epsilon^{p}$ in (10) and (11), we obtain the following set of linear equations in the coefficients $a_{n}^{p}, b_{n}^{p}(p \geq 0)$ :

$$
\begin{gathered}
\sum_{n=0}^{\infty} P_{n}(\eta)\left[\alpha_{n}^{1} a_{n}^{p}+n(n+1) \delta_{n}^{0} b_{n}^{p}\right]+\sum_{n=0}^{\infty} P_{n}(\eta) \sum_{a=1}^{p} \frac{\mathrm{C}^{a} f^{a}}{q !} \\
\cdot\left[\alpha_{n}^{a+1} a_{n}^{p-a}+n(n+1) \delta_{n}^{a} b_{n}^{p-a}-\frac{i u_{0}}{\alpha} i^{n}(2 n+1) \mu_{n}^{p+1} \delta_{p a}\right]=\mathrm{U} P_{1}(\eta) \delta_{p 0} \\
\sum_{n=0}^{\infty} P_{n}^{1}(\eta)\left[\gamma_{n}^{0} a_{n}^{p}+\left(\delta_{n}^{0}+\beta_{n}^{1}\right) b_{n}^{p}\right]+\sum_{n=0}^{\infty} P_{n}^{1}(\eta) \sum_{a=1}^{p} \frac{\mathrm{C}^{a} f^{a}}{q !} \\
\cdot\left[\gamma_{n}^{a} a_{n}^{p-a}+\left(\delta_{n}^{a}+\beta_{n}^{a+1}\right) b_{n}^{p-a}-\frac{i u_{0}}{\alpha} i^{n}(2 n+1) \nu_{r}^{p} \delta_{p a}\right]=\mathrm{U} P_{1}^{1}(\eta) \delta_{p 0},
\end{gathered}
$$

Multiplying Eq. (12) by $P_{n}(\eta)$, Eq. (13) by $P_{n}^{1}(\eta)$, and integrating the resulting equations from -1 to +1 with respect to $\eta$, one obtains for $p=0$ :

$$
\begin{aligned}
& \alpha_{n}^{1} a_{n}^{0}+n(n+1) \delta_{n}^{0} b_{n}^{0}-\frac{i u_{0}}{\alpha} i^{n}(2 n+1) \mu_{n}^{1}=\mathrm{U} \delta_{n 1} \\
& \gamma_{n}^{0} a_{n}^{0}+\left(\delta_{n}^{0}+\beta_{n}^{1}\right) b_{n}^{0}-\frac{i u_{0}}{\alpha} i^{n}(2 n+1) \nu_{n}^{0}=\mathrm{U} \delta_{n 1}
\end{aligned}
$$

and for $p>0$ :

$$
\begin{aligned}
& \alpha_{n}^{1} a_{n}^{p}+n(n+1) \delta_{n}^{0} b_{n}^{p} \\
& \quad+\sum_{m=0}^{\infty} \sum_{a=1}^{p} \frac{\mathrm{C}^{a}}{q !}\left[\alpha_{m}^{a+1} a_{m}^{p-a}+m(m+1) \delta_{m}^{a} b_{m}^{p-a}-\frac{i u_{0}}{\alpha} i^{m}(2 m+1) \mu_{m}^{a+1} \delta_{p a}\right] I_{m n}^{a}=0, \\
& \gamma_{n}^{0} a_{n}^{p}+\left(\delta_{n}^{0}+\beta_{n}^{1}\right) b_{n}^{p} \\
& \quad+\sum_{m=0}^{\infty} \sum_{a=1}^{p} \frac{\mathrm{C}^{a}}{q !}\left[\gamma_{m}^{a} a_{m}^{p-a}+\left(\delta_{m}^{a}+\beta_{m}^{a+1}\right) b_{m}^{p-a}-\frac{i u_{0}}{\alpha} i^{m}(2 m+1) \nu_{m}^{a} \delta_{p a}\right] J_{m n}^{a}=0,
\end{aligned}
$$

where

$$
\begin{aligned}
& I_{m n}^{a}=\frac{2 n+1}{2} \int_{-1}^{1} f^{a} P_{m}(\eta) P_{n}(\eta) d \eta \\
& J_{m n}^{a}=\frac{2 n+1}{2 n(n+1)} \int_{-1}^{1} f^{a} P_{m}^{1}(\eta) P_{n}^{1}(\eta) d_{n}, \quad J_{m n}^{a}=0 \quad \text { if } \quad m=0 \quad \text { or, } \quad n=0 .
\end{aligned}
$$

Eq. (14) determines the coefficients $a_{n}^{0}, b_{n}^{0}$ and once these are known, Eqs. (15) determine $a_{n}^{p}, b_{n}^{p}(p \geq 1)$ recursively in terms of $a_{n}^{p-a}, b_{p-a}^{n}(q=1, \cdots, p)$. 
In order to derive explicit expressions for $a_{n}^{p}, b_{n}^{p}$, let us write

$$
\begin{aligned}
& a_{n}^{p}=\left(i u_{0} / \alpha\right) A_{n}^{p}+U \mathrm{~A}_{n}^{p}, \\
& b_{n}^{p}=\left(i u_{0} / \alpha\right) B_{n}^{p}+U \mathrm{~B}_{n}^{p} .
\end{aligned}
$$

Thus, if $A_{n}^{p}$ and $B_{n}^{p}$ are used in place of $a_{n}^{p}, b_{n}^{p}$, respectively, in (7) the resulting $\chi$, $\psi$ give the scattered field when $B$ is kept fixed. The terms arising from $A_{n}^{p}, B_{n}^{p}$ give the radiated field from the oscillating inclusion.

Using (16) in (14)-(15), we get for $p=0$

$$
\begin{aligned}
& A_{n}^{0}=i^{n}(2 n+1) \frac{D_{n}}{\Delta_{n}}, \quad B_{n}^{0}=i^{n}(2 n+1) \frac{i}{\alpha \mathrm{C}^{3} \Delta_{n}}, \\
& \mathrm{~A}_{n}^{0}=0, \quad B_{n}^{0}=0, \quad n \neq 1, \\
& \mathrm{~A}_{1}^{0}=\frac{1}{\Delta_{1}}\left[\frac{\partial}{\partial C} h_{1}(\beta \mathrm{C})-\frac{1}{C} h_{1}(\beta \mathrm{C})\right], \\
& \mathrm{B}_{1}^{0}=\frac{1}{\Delta_{1}}\left[\frac{\partial}{\partial C} h_{1}(\alpha \mathrm{C})-\frac{1}{C} h_{1}(\alpha \mathrm{C})\right],
\end{aligned}
$$

with

$$
\begin{aligned}
& \Delta_{n}=\frac{\partial}{\partial \mathrm{C}} h_{n}(\alpha \mathrm{C})\left[\frac{\partial}{\partial C} h_{n}(\beta \mathrm{C})+\frac{1}{C} h_{n}(\beta \mathrm{C})\right]-\frac{n(n+1)}{\mathrm{C}^{2}} h_{n}(\alpha \mathrm{C}) h_{n}(\beta \mathrm{C}), \\
& D_{n}=\frac{\partial}{\partial \mathrm{C}} j_{n}(\alpha \mathrm{C})\left[\frac{\partial}{\partial C} h_{n}(\beta \mathrm{C})+\frac{1}{C} h_{n}(\beta \mathrm{C})\right]-\frac{n(n+1)}{\mathrm{C}^{2}} j_{n}(\alpha \mathrm{C}) h_{n}(\beta \mathrm{C}) .
\end{aligned}
$$

For $p>0$ we get

$$
\begin{aligned}
& A_{n}^{p}=\frac{1}{\Delta_{n}} \sum_{m=0}^{\infty} \sum_{q=1}^{p} \frac{\mathrm{C}^{q}}{q !}\left[n(n+1) \delta_{n}^{0} \mathcal{L}_{m, n}^{p \cdot Q}-\left(\delta_{n}^{0}+\beta_{n}^{1}\right) \Re_{m, n}^{p \cdot Q}\right], \\
& B_{n}^{p}=-\frac{1}{\Delta_{n}} \sum_{m=0}^{\infty} \sum_{a=1}^{p} \frac{\mathrm{C}^{Q}}{q !}\left[\alpha_{n}^{1} \mathcal{L}_{m, n}^{p, a}-\gamma_{n}^{0} \mathfrak{N T}_{m, n}^{p, Q}\right] \text {, }
\end{aligned}
$$

with

$$
\begin{aligned}
& \mathcal{L}_{m, n}^{\mathcal{p} \cdot q}=J_{m n}^{a}\left[\gamma_{m}^{a} A_{m}^{p-Q}+\left(\delta_{m}^{q}+\beta_{m}^{a+1}\right) B_{m}^{p-Q}-i^{m}(2 m+1) \nu_{m}^{q} \delta_{p q}\right], \\
& \mathfrak{N}_{m, n}^{p, q}=I_{m n}^{a}\left[\alpha_{m}^{q+1} A_{m}^{p-q}+m(m+1) \delta_{m}^{q} B_{m}^{p-q}-i^{m}(2 m+1) \mu_{m}^{q+1} \delta_{p q}\right] .
\end{aligned}
$$

Expressions for $\mathrm{A}_{n}^{p}, \mathrm{~B}_{n}^{p}$ are obtained from $A_{n}^{p}, B_{n}^{p}$, respectively, on replacing $A_{m}^{p-a}$, $B_{m}^{p-a}$ by $\mathrm{A}_{m}^{p-a}, \mathrm{~B}_{m}^{p-a}$, respectively, and omitting the last terms within brackets in (20). These expressions were derived in our earlier paper [14]. This completes the determination of the perturbation coefficients. It may be noted here that if $f(\theta)$ is a polynomial in $\sin \theta(\cos \theta)$, the series over $m$ will contain a finite number of terms. This will be clearly seen in the applications considered in the following section.

3. Response of the inclusion. Here we shall derive the expression for $U$ in terms of the amplitude $u_{0}$ of the incident displacement field. For this purpose we assume that 
$p_{1}$ is the density of the inclusion and $\mathrm{V}$ its volume. Then the equation of motion of $B$ gives $-\rho_{1} \mathrm{~V} \omega^{2} \mathrm{U}=\int_{-1}^{1} 2 \pi \mathrm{C}^{2}$

$$
\cdot\left[(1+\epsilon f)\left(\sigma_{r r} \cos \theta-\sigma_{r \theta} \sin \theta\right)+\epsilon f^{\prime}\left(\sigma_{\theta \theta} \sin \theta-\sigma_{r \theta} \cos \theta\right)\right](1+\epsilon f) d \eta .
$$

Here $\sigma_{r r}, \sigma_{r \theta}, \sigma_{\theta \theta}$ are the time-independent parts of the stresses $\tau_{r r}, \tau_{r \theta}, \tau_{\theta \theta}$, evaluated at $r=\mathrm{C}(1+\epsilon f)$. These are

$$
\begin{aligned}
\frac{\sigma_{r r}}{2 \mu}= & \sum_{n=0}^{\infty} P_{n}(\eta)\left[a_{n}\left(\frac{d^{2}}{d r^{2}} h_{n}(\alpha r)-\frac{\alpha^{2} \sigma}{1-2 \sigma} h_{n}(\alpha r)\right\}+n(n+1) b_{n} \frac{d}{d r}\left(\frac{h_{n}(\beta r)}{r}\right)\right. \\
& \left.-\frac{i u_{0}}{\alpha} i^{n}(2 n+1)\left\{\frac{d^{2}}{d r^{2}} j_{n}(\alpha r)-\frac{\alpha^{2} \sigma}{1-2 \sigma} j_{n}(\alpha r)\right\}\right]_{r-\mathrm{c}(1+\epsilon)}, \\
\frac{\sigma_{r \theta}}{\mu}= & -\sum_{n=0}^{\infty} P_{n}^{1}(\eta)\left[2 a_{n} \frac{d}{d r}\left(\frac{h_{n}(\alpha r)}{r}\right)+b_{n}\left\{\frac{d^{2}}{d r^{2}} h_{n}(\beta r)+\frac{(n+2)(n-1)}{r^{2}} h_{n}(\beta r)\right\}\right. \\
& \left.-\frac{i u_{0}}{\alpha} 2 i^{n}(2 n+1) \frac{d}{d r}\left\{\frac{j_{n}(\alpha r)}{r}\right\}\right]_{r-\mathrm{c}(1+\epsilon)}, \\
\frac{\sigma_{\theta \theta}}{2 \mu}= & \sum_{n=0}^{\infty}\left[a_{n}\left\{-\frac{1}{r^{2}} h_{n}(\alpha r) \frac{d P_{n}^{1}}{d \theta}+\left(\frac{1}{r} \frac{d}{d r}\left(h_{n}(\alpha r)\right)-\frac{\alpha^{2} \sigma}{1-2 \sigma} h_{n}(\alpha r)\right) P_{n}\right\}\right. \\
& +b_{n} \frac{1}{r^{2}}\left\{-\frac{d}{d r}\left(r h_{n}(\beta r)\right) \frac{d P_{n}^{1}}{d \theta}+n(n+1) h_{n}(\beta r) P_{n}\right\} \\
& \left.-\frac{i u_{0}}{\alpha} i^{n}(2 n+1)\left\{-\frac{1}{r^{2}} j_{n}(\alpha r) \frac{d P_{n}^{1}}{d \theta}+\left(\frac{1}{r} j_{n}(\alpha r)-\frac{\alpha^{2} \sigma}{1-2 \sigma} j_{n}(\alpha r)\right) P_{n}\right\}\right]_{r-\mathrm{c}(1+\sigma r)} .
\end{aligned}
$$

Using (22) in (21) we obtain, after some lengthy calculations,

$$
\mathrm{U}=-\frac{i u_{0}}{\alpha} \frac{F}{\mathrm{~F}+\frac{1}{4 \pi \bar{\rho}} \mathrm{V} \beta^{2}}, \quad \bar{\rho}=\rho_{1} / \rho
$$

where

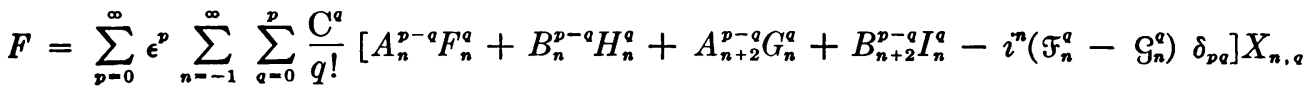

$$
\begin{aligned}
& +\sum_{p=0}^{\infty} \epsilon^{p+1} \sum_{n=0}^{\infty} \sum_{q=0}^{p} \frac{\mathrm{C}^{Q}}{q !}\left[A_{n}^{p-q} f_{n}^{Q}+B_{n}^{p-Q} h_{n}^{Q}+A_{n+2}^{p-q} g_{n}^{Q}+B_{n+2}^{p-q} i_{n}^{Q}-i^{n}\left(w_{n}^{Q}-z_{n}^{Q}\right) \delta_{p Q}\right] Y_{n, Q} \text {, } \\
& X_{n, Q}=\int_{-1}^{1} f^{a} P_{n+1}(\eta) d \eta, \quad Y_{n, a}=\int_{-1}^{1} f^{\prime a} f^{1} P_{n+1}(\eta) d \eta .
\end{aligned}
$$

The expressions for $F_{n}^{a}, f_{n}^{a}$, etc., are given in the Appendix A. The expression for $\mathrm{F}$ is obtained from $F$ on replacing $A_{n}^{p-a}$, etc., by $\mathrm{A}_{n}^{p-a}$ and omitting the last terms within the square brackets in Eq. (24). Thus $\mathrm{U}$ can be determined to any desired $O\left(\epsilon^{p}\right)$. In the following subsections we shall confine our attention to calculating $\mathrm{U}$ correct to $O(\epsilon)$ when $\mathrm{S}$ is either an oblate or a prolate spheroid. 
1.3 Oscillations of an oblate spheroidal inclusion. When the surface $\mathrm{S}$ is an oblate spheroid of eccentricity $e$ and semi-major axis $a$, its equation is

$$
r=a\left(1-e^{2}\right)^{1 / 2} /\left(1-e^{2} \sin ^{2} \theta\right)^{1 / 2} .
$$

We shall assume that $e \neq 1$. Then (26) can be put in the form (3) by choosing

$$
\begin{aligned}
\mathrm{C} & =\frac{a}{2}\left[1+\left(1-e^{2}\right)^{1 / 2}\right], \quad \epsilon=\left(1-\left(1-e^{2}\right)^{1 / 2}\right) /\left(1+\left(1-e^{2}\right)^{1 / 2}\right), \\
\epsilon f(\theta) & =\frac{1-\epsilon^{2}}{\left(1+\epsilon^{2}+2 \epsilon \cos 2 \theta\right)^{1 / 2}}-1=\epsilon\left(2 \sin ^{2} \theta-1\right)+O\left(\epsilon^{2}\right) .
\end{aligned}
$$

Thus correct to $O(\epsilon)$ the equation of $\mathrm{S}$ may be written as

$$
r=\mathrm{C}\left[1+\epsilon\left(2 \sin ^{2} \theta-1\right)\right], \quad f(\theta)=2\left(\sin ^{2} \theta-\frac{1}{2}\right) .
$$

Using this value of $f(\theta)$ in (24) one obtains $X_{n, q}, Y_{n, q}$. Since we are interested in calculating $F, \mathrm{~F}$ correct to $O(\epsilon)$, we only need the values of $X_{n, 0}, X_{n, 1}, Y_{n, 0}$, and these are

$$
\begin{aligned}
X_{n, 0} & =0, & & n \neq-1, \\
& =2, & & n=-1, \\
X_{n, 1} & =2 / 3, & & n=-1, \\
& =-8 / 15, & & n=1, \\
& =0, & & n \neq-1,1, \\
Y_{n, 0} & =0, & & n \neq 1, \\
& =16 / 5, & & n=1,
\end{aligned}
$$

Thus from (24) and (A.1)-(A.3) we obtain, after some simplifications,

$$
\begin{aligned}
F=-\frac{1}{3} \beta^{2} \mathrm{C}^{2}\left[-3 i j_{1}\right. & +A_{1}^{0} h_{1}(\alpha \mathrm{C})+2 B_{1}^{0} h_{1}(\beta \mathrm{C}) \\
& +\epsilon\left\{A_{1}^{1} h_{1}(\alpha \mathrm{C})+2 B_{1}^{1} h_{1}(\beta \mathrm{C})+\frac{1}{5} A_{1}^{0}\left(-\alpha \mathrm{C} h_{0}(\alpha \mathrm{C})+8 h_{1}(\alpha \mathrm{C})\right)\right. \\
& +\frac{1}{5} B_{1}^{0}\left(6 \beta \mathrm{C} h_{0}(\beta \mathrm{C})-8 h_{1}(\beta \mathrm{C})\right)-\frac{3 i}{5}\left(8 j_{1}(\alpha \mathrm{C})-\alpha C j_{0}(\alpha \mathrm{C})\right) \\
& \left.\left.-3\left(\frac{4}{35} A_{3}^{0} \alpha \mathrm{C} h_{2}(\alpha \mathrm{C})+\frac{16}{35} B_{3}^{0} \beta C h_{2}(\beta \mathrm{C})+i \alpha \mathrm{C} \frac{4}{5} j_{2}(\alpha \mathrm{C})\right)\right\}\right] .
\end{aligned}
$$

Similarly, $\mathbf{F}$ can be written as

$$
\begin{aligned}
\mathrm{F}=-\frac{1}{3} \beta^{2} \mathrm{C}^{2}\left[\bar{A}_{1}^{0} h_{1}(\alpha \mathrm{C})\right. & +2 \bar{B}_{1}^{0} h_{1}(\beta \mathrm{C}) \\
+ & \epsilon\left\{\bar{A}_{1}^{1} h_{1}(\alpha \mathrm{C})+2 \bar{B}_{1}^{1} h_{1}(\beta \mathrm{C})+\frac{1}{5} \bar{A}_{1}^{0}\left(-\alpha \mathrm{C} h_{0}(\alpha \mathrm{C})+8 h_{1}(\alpha \mathrm{C})\right)\right. \\
& \left.\left.+\frac{1}{5} \bar{B}_{1}^{0}\left(6 \beta \mathrm{C} h_{0}(\beta \mathrm{C})-8 h_{1}(\beta \mathrm{C})\right)\right\}\right] .
\end{aligned}
$$

This last expression agrees with the corresponding result obtained in our earlier paper ([14], Eqs. (47)-(49)). Use of (29), (30) in (23) gives U. 
For a sphere of radius $\mathrm{C}, \mathrm{V}=\frac{4}{3} \pi \mathrm{C}^{3}$, and $\mathrm{U}$ can be obtained by first putting $\epsilon=0$ in (29), (30), and using the resulting expressions for $F, \mathrm{~F}$ in (23). We get

$$
\mathrm{U}=-\frac{i u_{0}}{\alpha} \frac{3 \bar{\rho} \beta / \alpha}{C \bar{\Delta}} h_{2}(\beta \mathrm{C})
$$

This checks with the result derived by Mow [2]. Here

$$
\bar{\Delta}=-\beta \mathrm{C}(1-\bar{\rho}) h_{1}(\alpha \mathrm{C}) h_{2}(\beta \mathrm{C})-2(1-\bar{\rho}) \alpha \mathrm{C} h_{1}(\beta \mathrm{C}) h_{2}(\alpha C)+\alpha \beta \mathrm{C}^{2} h_{2}(\alpha \mathrm{C}) h_{2}(\beta \mathrm{C})
$$

In the following we shall derive low and high jrequency expansions for $F, \mathrm{~F}$, and $\mathrm{U}$.

3.1a Low-frequency expansion. First we shall obtain expressions for $F$ and $F$ correct to $O(\alpha \mathrm{C})$. These then can be checked with those derived by the integral equation method used by Williams [19] and Lawrence [17]. This latter method gives in a very simple and straightforward manner expressions for $F, \mathrm{~F}$ correct to $O(\alpha \mathrm{C})$. However, it does not give higher-order terms, whereas Eqs. (29) and (30) are valid for all frequencies as long as $\alpha \mathrm{C} \epsilon$ is small. To obtain the low-frequency expansions from (29), (30) correct to $O(\alpha \mathrm{C})$ we first note that

$$
\begin{aligned}
& A_{1}^{0}=i \alpha \mathrm{A}_{1}^{0}+O\left(\alpha^{3} \mathrm{C}^{3}\right), \\
& B_{1}^{0}=i \alpha \mathrm{B}_{1}^{0}+O\left(\alpha^{3} \mathrm{C}^{3}\right), \\
& A_{3}^{0}=O\left(\alpha^{5} \mathrm{C}^{5}\right), \quad B_{3}^{0}=O\left(\alpha^{5} \mathrm{C}^{5}\right) .
\end{aligned}
$$

Then from (19) for $p=1, n=1$ we find that correct to $O(\alpha \mathrm{C})$

$$
A_{1}^{1} \approx \mathrm{A}_{1}^{1}, \quad B_{1}^{1} \approx \mathrm{B}_{1}^{1} .
$$

Thus we arrive at the result

$$
F=i \alpha \mathrm{F}+O\left(\alpha^{3} \mathrm{C}^{3}\right) .
$$

This agrees with the general result derived in [17]. In [14] we derived a low-frequency expansion for $F$ correct to $O(\alpha \mathrm{C})$ that was shown to check with that derived by the method of [19]. We obtained

$$
\begin{aligned}
\mathrm{F} \approx-\frac{3 \mathrm{C}}{q^{2}+2}[1+ & \left.\epsilon \frac{12}{5} \frac{1-q^{2} / 6}{q^{2}+2}\right] \\
& \cdot\left[1-i \beta \mathrm{C} \frac{q^{3}+2}{q^{2}+2}\left(1+\epsilon \frac{12}{5} \frac{1-q^{2} / 6}{q^{2}+2}\right)\right], \quad q=\alpha / \beta .
\end{aligned}
$$

Eq. (31) now gives

$$
\mathrm{U}=u_{0}+O\left(\alpha^{2} \mathrm{C}^{2}\right) .
$$

This shows that at very low frequencies (so that terms of $O\left(\alpha^{2} \mathrm{C}^{2}\right)$ may be neglected) the amplitude of oscillation of the inclusion is independent of the shape of the inclusion and is the same as the amplitude of the incident field.

3.1b High-frequency expansion. For large $\alpha \mathrm{C}$ we find that

$$
\mathrm{F} \sim \frac{2}{3} i \beta \mathrm{C}^{2}\left[1+\beta / \alpha-\frac{2}{5} \epsilon\left(1-\frac{11 \beta}{4 \alpha}\right)\right] .
$$


It is interesting to note from the above expression that for large $\beta \mathrm{C}$ the time-independent part of the force necessary to maintain the steady oscillation of the spheroid along its axis is $O(\beta \mathrm{C})$. This result is to be compared with that obtained by us [15] for the torque necessary to maintain steady angular oscillations of the spheroid about its axis. There we calculated the torque correct to $O\left(\epsilon^{3}\right)$ and found that it was of $O(\beta C)$ for large $\beta C$. This suggests that if we had calculated $\bar{F}$ keeping higher powers of $\epsilon$ we would obtain

$$
\mathrm{F} \sim \frac{2}{3} i \beta \mathrm{C}^{2}\left[\sum_{n=0}^{p} a_{n} \epsilon^{n}+O\left(\epsilon^{p+1}\right)\right]
$$

where the coefficients $a_{n}$ are independent of the frequency of oscillation of the spheroid. However, we have been unable to prove this result without carrying out the tedious calculations for obtaining the coefficients of different powers of $\epsilon$.

For $F$ we find

$$
F \sim-i \frac{\beta^{2}}{\alpha} \mathrm{C}\left[1+i \alpha \mathrm{C} \epsilon\left(1+\frac{2 \alpha}{\beta}\right)\right]
$$

provided $\alpha \mathrm{C} \epsilon$ is very small. Using (39) and (37) in (31) we obtain

$$
\mathrm{U} \sim-u_{0} \frac{4 \pi \bar{\rho} \mathrm{C}}{\alpha^{2} V} \exp (-i \alpha \mathrm{C})\left[1+i \alpha \mathrm{C} \epsilon\left(1+\frac{2 \alpha}{\beta}\right)\right]
$$

For the sphere $\epsilon=0$ and one recovers the result derived in [2].

3.2 Oscillations of a prolate spheroidal inclusion. If the surface $S$ is a prolate spheroid, then its equation is

$$
r=a\left(1-e^{2}\right)^{1 / 2} /\left(1-e^{2} \cos ^{2} \theta\right)^{1 / 2},
$$

$a, e$ having the same meaning as in (26). It is easily shown that all the results for this case can be obtained from the previous subsection (3.1) on changing $\epsilon$ to $-\epsilon$. So in the next two sections we shall confine our attention to an oblate spheroid.

4. Diffraction by an oblate spheriod. For the analysis in this and the following section we shall assume $B$ to be fixed. In this section we shall obtain the leading terms in the asymptotic expansion for high frequencies of the potentials $\chi$ and $\psi$ valid in the illuminated region. We shall confine our attention to obtaining these expansions correct to $O(\alpha \mathrm{C} \epsilon)$. The method is a standard one used in [22,23]. For the purpose of illustration we shall only consider the region away from the shadow boundary and the caustics. The field in these latter regions can be obtained by the usual methods (see [24]).

Let

$$
\chi=\chi_{0}+\epsilon \chi_{1}, \quad \psi=\psi_{0}+\epsilon \psi_{1},
$$

with

$$
\chi_{0}=\frac{i u_{0}}{\alpha} \sum_{n=0}^{\infty} A_{n}^{0} h_{n}(\alpha r) P_{n}(\eta), \quad \frac{\partial \psi_{0}}{\partial \theta}=\frac{i u_{0}}{\alpha} \sum_{n=0}^{\infty} B_{n}^{0} h_{n}(\beta r) \frac{d P_{n}}{d \theta}
$$

and

$$
\chi_{1}=\frac{i u_{0}}{\alpha} \sum_{n=0}^{\infty} A_{n}^{1} h_{n}(\alpha r) P_{n}(\eta), \quad \frac{\partial \psi_{1}}{\partial \theta}=\frac{i u_{0}}{\alpha} \sum_{n=0}^{\infty} B_{n}^{1} h_{n}(\beta r) \frac{d P_{n}}{d \theta}
$$


Here $A_{n}^{0}$ and $B_{n}^{0}$ are given by (17). For $f(\theta)$ given by $(28)_{2}$ it is easily shown that

$$
\begin{aligned}
& I_{m n}^{1}=C_{2}(n) \delta_{m, n+2}+C_{0}(n) \delta_{m, n}+C_{-2}(n) \delta_{m, n-2}, \\
& J_{m n}^{1}=d_{2}(n) \delta_{m, n+2}+d_{0}(n) \delta_{m, n}+d_{-2}(n) \delta_{m, n-2}
\end{aligned}
$$

where $C_{2}(n)$, etc., are given in Appendix B. It is to be noted that for large $n$

$$
\mathrm{C}_{2} \sim \mathrm{C}_{-2} \sim d_{2} \sim d_{-2} \sim-\frac{1}{2}, \quad \mathrm{C}_{0} \sim d_{0} \sim 0 .
$$

Also, note that

$$
\mathrm{C}_{2}(-n-1)=\mathrm{C}_{-2}(n), \quad d_{2}(-n-1)=d_{-2}(n) .
$$

From (19) ${ }_{1}$ for $p=1$ we then obtain

$$
\begin{aligned}
A_{n}^{1}= & \frac{i}{\alpha \mathrm{C}} \sum_{s=-1}^{1} \frac{i^{n+2 s}(2 n+4 s+1)}{\bar{\Delta}_{n} \bar{\Delta}_{n+2 s}}\left[n ( n + 1 ) \frac { h _ { n } ( \beta \mathrm { C } ) } { \beta \mathrm { C } } d _ { 2 s } \left\{\frac{1}{\alpha^{2} \mathrm{C}^{2}}\left(h_{n+2 s}^{\prime}(\beta \mathrm{C})+\frac{1}{\beta C} h_{n+2 s}(\beta \mathrm{C})\right)\right.\right. \\
& \left.-\frac{(n+2 s)(n+2 s+1)}{\alpha^{2} \beta \mathrm{C}^{3}} h_{n+2 s}(\beta \mathrm{C})\right\} \\
& -\left(\frac{1}{\beta \mathrm{C}} h_{n}(\beta \mathrm{C})+h_{n}^{\prime}(\beta \mathrm{C})\right) C_{2 s}\left\{\frac{2(n+2 s)(n+2 s+1)}{\alpha^{2} \beta \mathrm{C}^{3}} h_{n+2 s}(\beta \mathrm{C})\right. \\
& \left.+\left(1-\frac{(n+2 s)(n+2 s+1)}{\alpha^{2} \mathrm{C}^{2}}\right)\left(\frac{1}{\beta \mathrm{C}} h_{n+2 s}(\beta \mathrm{C})+h_{n+2 s}^{\prime}(\beta \mathrm{C})\right)\right\} \\
& +\frac{\beta}{\alpha^{2} \mathrm{C}}\left\{n(n+1) d_{2 s} \frac{1}{\beta \mathrm{C}} h_{n}(\beta \mathrm{C})\left(h_{n+2 s}^{\prime \prime}(\beta \mathrm{C})+\left(\frac{1}{\beta \mathrm{C}} h_{n+2 s}(\beta \mathrm{C})\right)^{\prime}\right)\right. \\
& \left.\left.-(n+2 s)(n+2 s+1) C_{2 s}\left(\frac{1}{\beta \mathrm{C}} h_{n}(\beta \mathrm{C})+h_{n}^{\prime}(\beta \mathrm{C})\right)\left(\frac{1}{\beta \mathrm{C}} h_{n+2 s}(\beta \mathrm{C})\right)^{\prime}\right\}\right] .
\end{aligned}
$$

Here $\bar{\Delta}_{n}=(1 / \alpha \beta) \Delta_{n}$ and the primes denote derivatives with respect to the arguments. Similarly, from $(19)_{2}$ we shall get

$$
\begin{aligned}
B_{n}^{1}= & \frac{i}{\beta \mathrm{C}} \sum_{s=-1}^{1} \frac{i^{n+2 s}(2 n+4 s+1)}{\bar{\Delta}_{n} \bar{\Delta}_{n+2 s}}\left[h _ { n } ^ { \prime } ( \alpha \mathrm { C } ) d _ { 2 s } \left\{\frac{1}{\alpha^{2} \mathrm{C}^{2}}\left(h_{n+2 s}^{\prime}(\beta \mathrm{C})+\frac{1}{\beta \mathrm{C}} h_{n+2 s}(\beta \mathrm{C})\right)\right.\right. \\
& \left.-\frac{(n+2 s)(n+2 s+1)}{\alpha^{2} \beta \mathrm{C}^{3}} h_{n+2 s}(\beta \mathrm{C})\right\} \\
& -\frac{1}{\alpha \mathrm{C}} h_{n}(\alpha \mathrm{C}) C_{2 s}\left\{\frac{2(n+2 s)(n+2 s+1)}{\alpha^{2} \beta \mathrm{C}^{3}} h_{n+2 s}(\beta \mathrm{C})\right. \\
& \left.+\left(1-\frac{(n+2 s)(n+2 s+1)}{\alpha^{2} \mathrm{C}^{2}}\right)\left(h_{n+2 s}^{\prime}(\beta \mathrm{C})+\frac{1}{\beta \mathrm{C}} h_{n+2 s}(\beta \mathrm{C})\right)\right\} \\
& +\frac{\beta}{\alpha^{2} \mathrm{C}}\left\{d_{2 s} h_{n}^{\prime}(\alpha \mathrm{C})\left(h_{n+2 s}^{\prime \prime}(\beta \mathrm{C})+\left(\frac{1}{\beta \mathrm{C}} h_{n+2 s}(\beta \mathrm{C})\right)^{\prime}\right)\right. \\
& \left.\left.-C_{2 s}(n+2 s)(n+2 s+1) \frac{1}{\alpha \mathrm{C}} h_{n}(\alpha \mathrm{C})\left(\frac{1}{\beta \mathrm{C}} h_{n+2 s}(\beta \mathrm{C})\right)^{\prime}\right\}\right] .
\end{aligned}
$$

The series expressions (43), and (44), for $\chi_{0}, \psi_{0}, \chi_{1}, \psi_{1}$ are suitable for evaluating the field at any point when $\alpha \mathrm{C}$ is very small. However, these are useless when $\alpha \mathrm{C}$ is 
large. In the following, then, we shall obtain asymptotic expansions for these field quantities valid for large $\alpha \mathrm{C}(\alpha \mathrm{C} \gg 1, \alpha \mathrm{C} \epsilon \ll 1)$.

First, let us consider $\chi_{0}$. This is the dilatational potential for the scattered field due to a fixed rigid sphere of radius $\mathrm{C}$. We shall denote by $\bar{\chi}$ the total potential of the incident field given by (4) and the scattered field given by $\chi_{0}$. So

$$
\bar{\chi}=-\frac{i u_{0}}{\alpha} \sum_{n=0}^{\infty} i^{n}(2 n+1)\left[j_{n}(\alpha r)-\frac{D_{n}}{\Delta_{n}} h_{n}(\alpha r)\right] P_{n}(\eta) .
$$

This may alternatively be written as

$$
\bar{\chi}=-\frac{i u_{0}}{2 \alpha}\left(\frac{\pi}{2 \alpha r}\right)^{1 / 2} \sum_{n=0}^{\infty} i^{n}(2 n+1)\left[H_{n+1 / 2}^{(2)}(\alpha r)-\frac{\bar{D}_{n}}{\bar{\Delta}_{n}} H_{n+1 / 2}^{(1)}(\alpha r)\right] P_{n}(\eta)
$$

Here

$$
\begin{aligned}
\bar{D}_{n}=h_{n}^{(2) \prime}(\alpha \mathrm{C})\left[\frac{1}{\beta \mathrm{C}} h_{n}(\beta \mathrm{C})+h_{n}^{\prime}(\beta \mathrm{C})\right]-\frac{n(n+1)}{\alpha \beta \mathrm{C}^{2}} h_{n}^{(2)}(\alpha \mathrm{C}) h_{n}(\beta \mathrm{C}), & \\
& h_{n}^{(2)}(z)=(\pi / 2 z)^{1 / 2} H_{n+1 / 2}^{(2)}(z) .
\end{aligned}
$$

Using Watson's transformation we may write this as

$$
\begin{aligned}
\bar{\chi}=-\frac{i u_{0}}{2 \alpha}\left(\frac{\pi}{2 \alpha r}\right)^{1 / 2} & \exp (-i \pi / 4) \\
& \cdot \int_{\mathrm{C}_{\nu}} \frac{\nu \exp \left(-\frac{1}{2} i \pi \nu\right)}{\cos \pi \nu}\left[H_{\nu}^{(2)}(\alpha r)-\frac{\bar{D}_{\nu-1 / 2}}{\bar{\Delta}_{\nu-1 / 2}} H_{\nu}^{(1)}(\alpha r)\right] P_{\nu-1 / 2}(\eta) d \nu
\end{aligned}
$$

where the contour $C_{w}$ is shown in Fig. 1. Since

$$
P_{-\nu-1 / 2}(\eta)=P_{\nu-1 / 2}(\eta), \quad H_{-\nu}^{(1)}(z)=\exp (i \pi \nu) H_{\nu}^{(1)}(z), \quad H_{-\nu}^{(2)}(z)=\exp (-i \pi \nu) H_{\nu}^{(2)}(z),
$$

we see that the integrand is an odd function of $\nu$. So the lower half of the contour $C_{w}$ may be replaced by its reflection about the origin and (52) may be written as

$$
\begin{aligned}
\bar{\chi}=-\frac{i u_{0}}{\alpha}\left(\frac{\pi}{2 \alpha r}\right)^{1 / 2} \exp (-i \pi / 4) \sum_{m=0}^{\infty}(-1)^{m} \\
\cdot \int_{-\infty}^{\infty} \nu \exp (i \pi \nu(2 m+1 / 2))\left[H_{\nu}^{(2)}(\alpha r)-\frac{\bar{D}_{\nu-1 / 2}}{\bar{\Delta}_{\nu-1 / 2}} H_{\nu}^{(1)}(\alpha r)\right] P_{\nu-1 / 2}(\eta) d \nu .
\end{aligned}
$$

Now for large $\alpha \mathrm{C}$ the zeros of $\Delta_{\nu-1 / 2} q u a$ function of $\nu$ are approximately those of $H_{v}^{(1)}(\alpha \mathrm{C})$ and $H_{v}^{(1)}(\beta \mathrm{C})$, and the zeros of these with positive imaginary parts lie along the dashed curves $h_{1}, h_{2}$, respectively, shown in Fig. 1. The first set of zeros lying along $h_{1}$ is given by

$$
\nu_{1 n} \sim \alpha \mathrm{C}+x_{n}\left(\frac{\alpha \mathrm{C}}{2}\right)^{1 / 3} \exp (i \pi / 3), \quad n=1,2, \cdots
$$

where $-x_{n}$ is the $n$th zero of the Airy function $\operatorname{Ai}(x)$. Similarly, the second set of zeros lying along $h_{2}$ are

$$
\nu_{2 n} \sim \beta \mathrm{C}+x_{n}\left(\frac{\beta \mathrm{C}}{2}\right)^{1 / 3} \exp (i \pi / 3), \quad n=1,2, \cdots
$$


The next step is to reduce the integral in (53) to a sum of residues at the zeros of $\Delta_{,-1 / 2}$. In order to do this we close the contour in the upper half of the $\nu$-plane by a sequence of large semicircles $C_{n}$ of radius $R_{n}$ passing between the zeros (see Fig. 1).

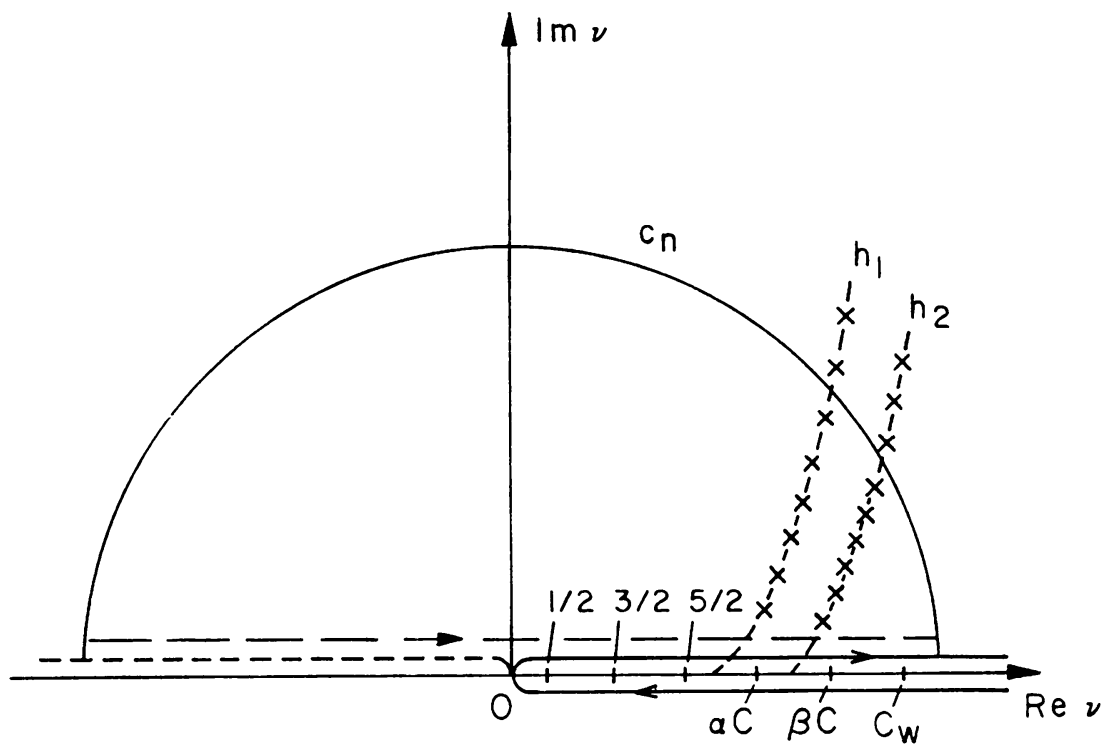

Fig. 1. Contour for Watson's transformation.

It can be shown in a manner similar to that employed in [24] that the contribution from the integral over $C_{n}$ tends to zero as $R_{n}$ tends to infinity for $0 \leq \theta<\pi$ when $m \neq 0$ and for $0<\theta<\pi / 2$ for $m=0$. So for $m=0$ we cannot replace the integral (53) by its residue series if $\theta>\pi / 2$. Now it is shown in [24] that $m=0$ term gives the dominant contribution in the illuminated region $\left(\theta>\theta_{0}=\sin ^{-1} \mathrm{C} / r\right)$. The same result can be shown to be true in the present case also. To obtain, then, the dominant terms in the illuminated region we shall evaluate the integral in (53) for $m=0$.

For $m=0$ we shall write

$$
\begin{aligned}
\bar{\chi}_{0}=-\frac{i u_{0}}{\alpha}\left(\frac{\pi}{2 \alpha r}\right)^{1 / 2} \exp (-i \pi / 4) \\
\quad \cdot \int_{-\infty}^{\infty} \nu \exp (i \pi \nu / 2) g(\nu, \alpha \mathrm{C}, \beta \mathrm{C}, \alpha r)\left[Q_{\nu-1 / 2}^{(1)}(\eta)+Q_{\nu-1 / 2}^{(2)}(\eta)\right] d \nu
\end{aligned}
$$

where $g(\nu, \alpha \mathrm{C}, \beta \mathrm{C}, \alpha r)$ stands for the expression within the square bracket in (53). $Q_{\nu-1 / 2}^{(1)}, Q_{\nu-1 / 2}^{(2)}$ are defined in [24] (Eq. (C.1)). The dominant contribution comes from the integral involving $Q_{v-1 / 2}^{(1)}$ as long as we are not close to the line $\theta=\pi$. We shall restrict our attention here in the region away from the line $\theta=\pi$, and away from the shadow boundary and from the surface of the sphere $r=\mathrm{C}$. The saddle-point approximation of the integral

$$
I=\int_{-\infty}^{\infty} \nu \exp (i \nu \pi / 2) g Q_{\nu-1 / 2}^{(1)} d \nu
$$


gives

$$
I=I^{(i)}+I^{(r)}
$$

where

$$
I^{(i)} \sim\left(\frac{2 \alpha r}{\pi}\right)^{1 / 2} \exp (i \alpha r \cos \theta+i \pi / 4)
$$

$I^{(r)} \sim\left(\frac{2 \alpha r}{\pi}\right)^{1 / 2}\left(\frac{\mathrm{C}^{2} \sin 2 \zeta}{4 s r \sin \theta}\right)^{1 / 2}\left[\frac{\sin \zeta \sin \lambda-\cos \zeta \cos \lambda}{\sin \zeta \sin \lambda+\cos \zeta \cos \lambda}\right] \exp \left(i \alpha\left(s-\frac{3}{2} \mathrm{C} \sin \zeta\right)\right)$

Here

$$
s=r \cos \bar{w}-\frac{\mathrm{C}}{2} \sin \zeta, \quad \alpha \cos \zeta=\beta \cos \lambda, \quad \zeta=\frac{1}{2}(\theta-\bar{w}) .
$$

The contribution $I^{(i)}$ comes from the saddle point $\nu=\alpha r \sin \overline{\bar{w}}=\alpha r \sin \theta$ and represents the direct ray reaching the observation point $P(r, \theta) . I^{(r)}$ comes from the saddle point $\nu=\alpha r \sin \bar{w}=\alpha \mathrm{C} \cos \zeta$ and represents the reflected $P$-wave reaching $P$ (see Fig. 2).

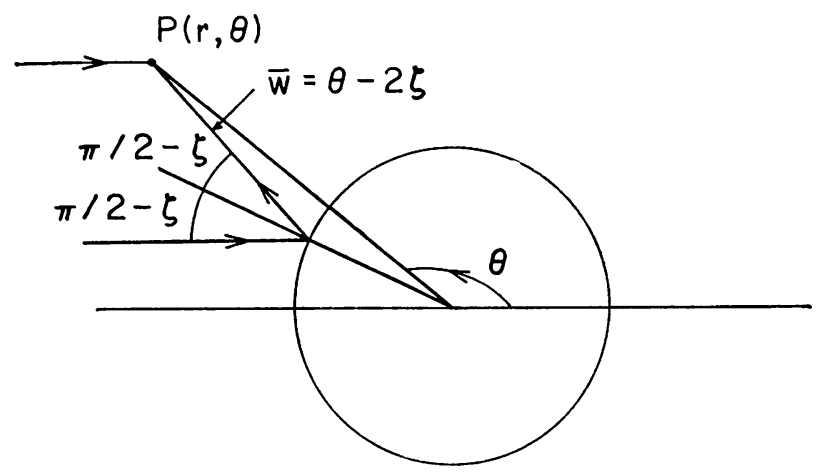

Fig. 2. P-reflected $\mathrm{P}$-wave from a rigid sphere.

Note that the expression within the square bracket in $(57)_{2}$ represents the reflection coefficient of the $P P$-reflected ray from a plane rigid boundary (see [25], Eq. (3-2)). So the first factor in (57) $)_{2}$ may be interpreted as the divergence coefficient of the $P P$ reflected wave. That this is true can be seen from the analysis in [20] for the reflection of a scalar wave off a totally-reflecting sphere (see Eq. (219)).

The reflected shear-wave potential can similarly be obtained by converting (4) into an integral of the form (53) and evaluating the dominant contribution from the $m=0$ term. We get

$$
\begin{aligned}
\frac{\partial \psi_{0}}{\partial \theta} \sim-\frac{i u_{0}}{\alpha}( & \left.\frac{\beta \mathrm{C}^{2} \sin ^{2} \lambda \cos \zeta}{r \sin \theta\left[\beta C \sin ^{2} \lambda+(\beta \sin \lambda+\alpha \sin \zeta)(r \cos W-\mathrm{C} \sin \lambda)\right]}\right)^{1 / 2} \\
& \cdot\left[\frac{2 \sin \zeta \cos \lambda}{\sin \zeta \sin \lambda+\cos \zeta \cos \lambda}\right] \\
& \cdot \exp (i[\beta(r \cos W-\mathrm{C} \sin \lambda)-\alpha \mathrm{C} \sin \zeta]), \quad W=\theta-\lambda-\zeta .
\end{aligned}
$$

This comes from the saddle point $\nu=\beta r \sin W=\beta \mathrm{C} \cos \lambda=\alpha \mathrm{C} \cos \zeta$ and represents the reflected $S$-wave reaching $P$ (see Fig. 3). Here the term within the square bracket 


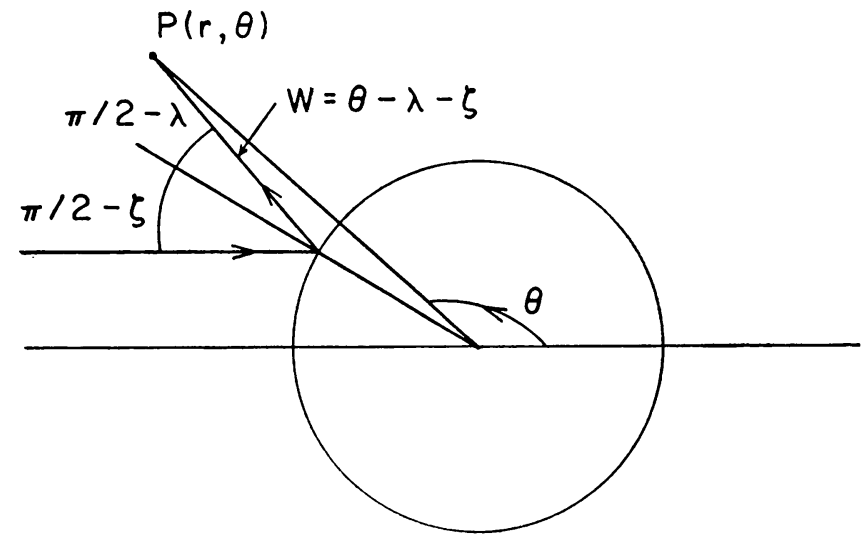

FIg. 3. P-reflected S-wave from a rigid sphere.

represents the reflection coefficient of $P S$-reflected ray from a rigid plane boundary ([25], Eq. (3-2)), the square root expression representing the divergence coefficient. In the next section we shall extend the ray theory of [20] to derive expressions for the divergence coefficients for the $P P$ - and $P S$-waves reflected off a rigid obstacle and show that they agree with what we have obtained above when the obstacle is a sphere.

We can evaluate in the same manner the dominant terms in the asymptotic expansions of the perturbation potentials for the reflected $P P$ - and $P S$-waves. We find

$$
\begin{gathered}
\chi_{1} \sim i \alpha \mathrm{C}(-2 \sin \zeta \cos 2 \zeta)\left[-\left(i u_{0} / \alpha\right)(\pi / 2 \alpha r)^{1 / 2} \exp (i \pi / 4) I^{(r)}\right], \\
\partial \psi_{1} / \partial \theta \sim-i \alpha \mathrm{C}(\sin \zeta+(\beta / \alpha) \sin \lambda) \cos 2 \zeta\left(\partial \psi_{0} / \partial \theta\right),
\end{gathered}
$$

where $\partial \psi_{0} / \partial \theta$ is given by (58). Thus the total reflected field (correct to $0(\alpha \mathrm{C} \epsilon)$ ) at the point $(r, \theta)$ are derived from the potentials

$\chi=\chi_{0}+\epsilon \chi_{1} \sim-\frac{i u_{0}}{\alpha} \frac{\sin \zeta \sin \lambda-\cos \zeta \cos \lambda}{\sin \zeta \sin \lambda+\cos \zeta \cos \lambda}\left(\frac{\mathrm{C}^{2} \sin 2 \zeta}{4 s r \sin \theta}\right)^{1 / 2}$

$$
\cdot \exp \left(i \alpha\left(s-\frac{3}{2} \mathrm{C} \sin \zeta\right)\right)[1-2 i \alpha \mathrm{C} \epsilon \sin \zeta \cos 2 \zeta]
$$

$\frac{\partial \psi}{\partial \theta}=\frac{\partial \psi_{0}}{\partial \theta}+\epsilon \frac{\partial \psi_{1}}{\partial \theta} \sim-\frac{i u_{0}}{\alpha} \frac{2 \sin \zeta \cos \lambda}{\sin \zeta \sin \lambda+\cos \zeta \cos \lambda}$

$$
\begin{aligned}
& \cdot\left(\frac{\beta \mathrm{C}^{2} \sin ^{2} \lambda \cos \zeta}{r \sin \theta\left[\beta \mathrm{C} \sin ^{2} \lambda+(\beta \sin \lambda+\alpha \sin \zeta)(r \cos W-\mathrm{C} \sin \lambda)\right]}\right)^{1 / 2} \\
& \cdot \exp (i \beta(r \cos W-\mathrm{C} \sin \lambda)-i \alpha \mathrm{C} \sin \zeta)\left[1-i \alpha \mathrm{C} \epsilon \cos 2 \zeta\left(\sin \zeta+\frac{\beta}{\alpha} \sin \lambda\right)\right] .
\end{aligned}
$$

We shall show in the next section that these results agree with those derived by the ray theory.

5. Diffraction by an arbitrary rigid surface of revolution. The analysis in this section is similar to that in [26] and follows closely that of [20]. In the illuminated region 
we have to compute the divergence coefficients for the $P$-reflected $P$ and $S$ waves to obtain the geometric solution. The divergence coefficient for the $P$-reflected $P$ is easily shown to be

$$
D_{p p}=\left[R_{1} r^{\prime} /\left(R_{1}+R\right) r \sin \theta\right]^{1 / 2}
$$

where $R_{1}$ is the radius of curvature of the curve of intersection of the reflected $P$-wave with the meridian plane through $Q$ at the point $Q, R=Q P$, and $r^{\prime}$ is the distance of $Q$ from the axis of symmetry (see Fig. 4). Similarly, the divergence coefficient for the $P$-reflected $S$ is

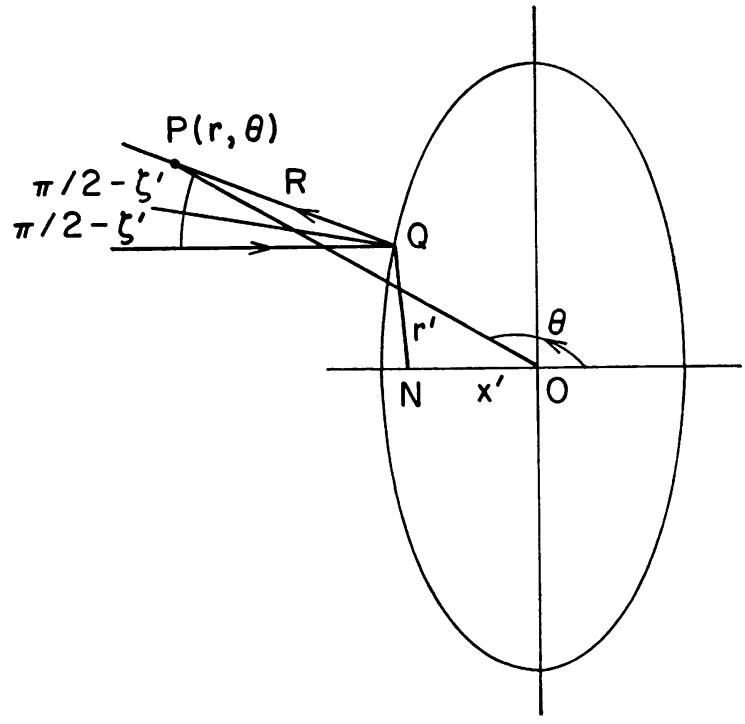

Fia. 4. P-reflected P-wave from an oblate spheroid when the wave is incident along its axis

$$
D_{p s}=\left[R_{2} r^{\prime \prime} /\left(R_{2}+R^{\prime}\right) r \sin \theta\right]^{1 / 2},
$$

$R_{2}$ being the radius of curvature of the curve of intersection of the reflected $S$-wave with the meridian plane through $Q^{\prime}$ at the point $Q^{\prime}, R^{\prime}=Q^{\prime} P$, and $r^{\prime \prime}$ is the distance of $Q^{\prime}$ from the axis (see Fig. 5).

To compute $R_{1}$ let us calculate the distance of two neighboring reflected $P$ rays on $S$. Let $\zeta^{\prime}$ be changed to $\zeta^{\prime}-\delta \zeta^{\prime}$. Then the elementary arc on $S$ is $\rho \delta \zeta^{\prime}$. The inclination of $R_{1}$ changes by $2 \delta \zeta^{\prime}$. Thus $R_{1} 2 \delta \zeta^{\prime}=\rho \delta \zeta^{\prime} \sin \zeta^{\prime}$. So

$$
R_{1}=\frac{1}{2} \rho \sin \zeta^{\prime}
$$

(see [20], Eq. (187)). Here $\rho$ is the radius of curvature of $S$ at $Q$. For the reflected $S$ rays we have $R_{2} \delta\left(\zeta^{\prime}+\lambda^{\prime}\right)=\rho^{\prime} \delta \zeta^{\prime} \sin \lambda^{\prime}$. Thus

$$
R_{2}=\rho^{\prime} \sin \lambda^{\prime} /\left(1+d \lambda^{\prime} / d \zeta^{\prime}\right) \text {. }
$$

We also have

$$
\cos \lambda^{\prime} / \cos \zeta^{\prime}=\alpha / \beta
$$




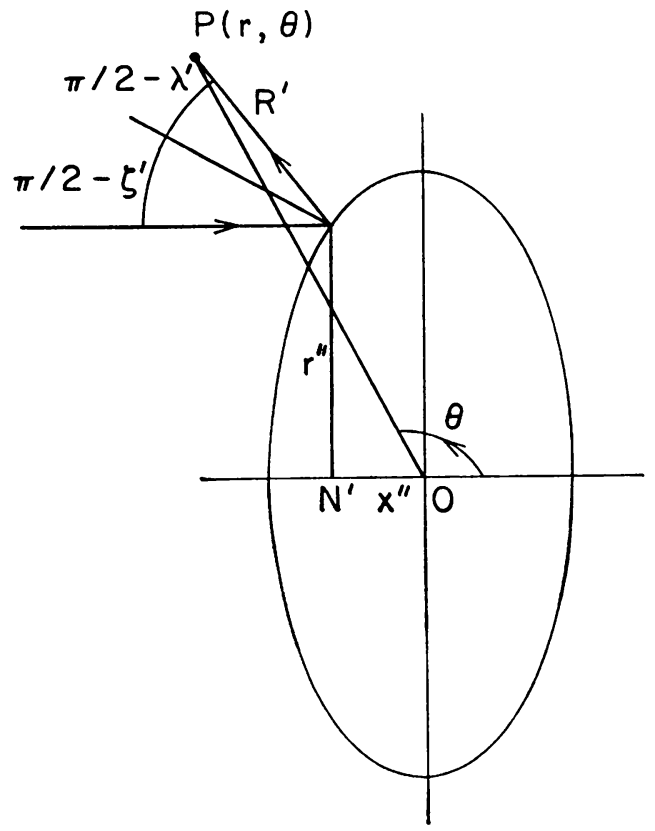

Fig. 5. P-reflected S-wave from the same spheroid as in Fig. 4.

So the reflected field is obtained from the potentials

$$
\begin{gathered}
\chi=-\frac{i u_{0}}{\alpha} \frac{\sin \zeta^{\prime} \sin \lambda^{\prime}-\cos \zeta^{\prime} \cos \lambda^{\prime}}{\sin \zeta^{\prime} \sin \lambda^{\prime}+\cos \zeta^{\prime} \cos \lambda^{\prime}}\left(\frac{\rho r^{\prime} \sin \zeta^{\prime}}{r \sin \theta\left(\rho \sin \zeta^{\prime}+2 R\right)}\right)^{1 / 2} \exp \left(i \alpha\left(R-x^{\prime}\right)\right), \\
\frac{\partial \psi}{\partial \theta}=-\frac{i u_{0}}{\alpha} \frac{2 \sin \zeta^{\prime} \cos \lambda^{\prime}}{\sin \zeta^{\prime} \sin \lambda^{\prime}+\cos \zeta^{\prime} \cos \lambda^{\prime}} \\
\cdot\left(\frac{\beta \rho r^{\prime \prime} \sin ^{2} \lambda^{\prime}}{r \sin \theta\left(\beta \rho \sin ^{2} \lambda^{\prime}+R^{\prime}\left(\beta \sin \lambda^{\prime}+\alpha \sin \zeta^{\prime}\right)\right)}\right)^{1 / 2} \exp \left(i \beta R^{\prime}-i \alpha x^{\prime \prime}\right)
\end{gathered}
$$

It is now easily shown that if the equation of $S$ is (28), then correct to $O(\alpha \mathrm{C} \epsilon)$ the expansions of $\chi, \partial \psi / \partial \theta$ in powers of $\epsilon$ agree with (61) and (62). For $R-x^{\prime}$ is then given by

$$
R-x^{\prime}=r \cos (\theta-2 \zeta)-2 \mathrm{C} \sin \zeta-2 \mathrm{C} \epsilon \sin \zeta \cos 2 \zeta+O\left(\epsilon^{2}\right),
$$

Similarly,

$$
\begin{aligned}
\beta R^{\prime}-\alpha x^{\prime \prime}=\beta r \cos (\theta-\lambda-\zeta)-\beta \mathrm{C} \sin \lambda & -\alpha C \sin \zeta \\
& -\mathrm{C} \epsilon(\beta \sin \lambda+\alpha \sin \zeta) \cos 2 \zeta+O\left(\epsilon^{2}\right) .
\end{aligned}
$$

Furthermore, we note that when $S$ is a sphere of radius $\mathrm{C}(68)$ and (69) agree with the expressions for $\chi_{0}, \partial \psi_{0} / \partial \theta$ given by (56)-(58). 
Appendix A. Here we give the expressions for $F_{n}^{p}$, etc.

$$
\begin{aligned}
F_{n}(r)= & \frac{n+1}{2 n+1}\left\{\frac{d^{2}}{d r^{2}} h_{n}(\alpha r)-\frac{\alpha^{2} \sigma}{1-2 \sigma} h_{n}(\alpha r)-n \frac{d}{d r}\left(\frac{h_{n}(\alpha r)}{r}\right)\right\} r^{2}, \\
G_{n}(r)= & \frac{n+2}{2 n+5}\left\{\frac{d^{2}}{d r^{2}} h_{n+2}(\alpha r)-\frac{\alpha^{2} \sigma}{1-2 \sigma} h_{n+2}(\alpha r)+(n+3) \frac{d}{d r}\left(\frac{h_{n+2}(\alpha r)}{r}\right)\right\} r^{2}, \\
H_{n}(r)= & \frac{n(n+1)}{2 n+1}\left\{(n+1) \frac{d}{d r}\left(\frac{h_{n}(\beta r)}{r}\right)-\frac{1}{2}\left(\frac{d^{2}}{d r^{2}} h_{n}(\beta r)+\frac{(n+2)(n-1)}{r^{2}} h_{n}(\beta r)\right)\right\} r^{2}, \\
I_{n}(r)= & \frac{(n+2)(n+3)}{2 n+5}\left\{(n+2) \frac{d}{d r}\left(\frac{h_{n+2}(\beta r)}{r}\right)\right. \\
& \left.+\frac{1}{2}\left(\frac{d^{2}}{d r^{2}} h_{n+2}(\beta r)+\frac{(n+1)(n+4)}{r^{2}} h_{n+2}(\beta r)\right)\right\} r^{2} .
\end{aligned}
$$

$(2 n+1)^{-1} \mathcal{F}_{n}(r)$ and $(2 n+5)^{-1} \mathcal{G}_{n}(r)$ are obtained from $F_{n}(r)$ and $G_{n}(r)$, respectively, on replacing $h_{n}(\alpha r)$, etc., by $j_{n}(\alpha r)$.

$$
\begin{aligned}
f_{n}(r)= & \frac{\mathrm{C}}{2 n+1}\left\{-\frac{n^{2}}{r} h_{n}(\alpha r)+\frac{d}{d r} h_{n}(\alpha r)+n r \frac{d}{d r}\left(\frac{h_{n}(\alpha r)}{r}\right)-\frac{\alpha^{2} \sigma}{1-2 \sigma} r h_{n}(\alpha r)\right\}, \\
g_{n}(r)= & \frac{\mathrm{C}}{2 n+5}\left\{\frac{(n+3)^{2}}{r} h_{n+2}(\alpha r)-\frac{d}{d r} h_{n+2}(\alpha r)\right. \\
& \left.+(n+3) r \frac{d}{d r}\left(\frac{h_{n+2}(\alpha r)}{r}\right)+\frac{\alpha^{2} \sigma}{1-2 \sigma} r h_{n+2}(\alpha r)\right\}, \\
h_{n}(r)= & \frac{\mathrm{C}}{2 n+1}\left\{-\frac{n^{2}}{r} \frac{d}{d r}\left(r h_{n}(\beta r)\right)+\frac{n(n+1)}{r} h_{n}(\beta r)\right. \\
& \left.+\frac{1}{2} n\left(r \frac{d^{2}}{d r^{2}} h_{n}(\beta r)+\frac{(n+2)(n-1)}{r} h_{n}(\beta r)\right)\right\}, \\
i_{n}(r)= & \frac{\mathrm{C}}{2 n+5}\left\{\frac{(n+3)^{2}}{r} \frac{d}{d r}\left(r h_{n+2}(\beta r)\right)-\frac{(n+2)(n+3)}{r} h_{n+2}(\beta r)\right. \\
& \left.+\frac{1}{2}(n+3)\left(r \frac{d^{2}}{d r^{2}} h_{n+2}(\beta r)+\frac{(n+4)(n+1)}{r} h_{n+2}(\beta r)\right)\right\} .
\end{aligned}
$$

$(2 n+1)^{-1} w_{n}(r)$ and $(2 n+5)^{-1} z_{n}(r)$ are obtained from $f_{n}(r)$ and $g_{n}(r)$, respectively, on replacing $h_{n}(\alpha r)$, etc. by $j_{n}(\alpha r)$.

$$
F_{n}^{p}=\left[\frac{d^{p}}{d r^{p}} F_{n}(r)\right]_{r=\mathrm{C}}
$$

Similarly, one obtains $G_{n}^{p}$, etc.

Appendix B.

$$
\begin{aligned}
I_{m n}^{1} & =-\int_{-1}^{1} \frac{2 n+1}{2} P_{m}(\eta) P_{n}(\eta)\left(2 \eta^{2}-1\right) d \eta \\
& =\mathrm{C}_{2}(n) \delta_{m, n+2}+\mathrm{C}_{0}(n) \delta_{m, n}+\mathrm{C}_{-2}(n) \delta_{m, n-2}
\end{aligned}
$$




$$
\begin{aligned}
\mathrm{C}_{2}(n) & =-\frac{2(n+1)(n+2)}{(2 n+3)(2 n+5)}, \quad \mathrm{C}_{-2}(n)=-\frac{2 n(n-1)}{(2 n-3)(2 n-1)} \\
\mathrm{C}_{0}(n) & =1-\frac{2}{2 n+1}\left(\frac{(n+1)^{2}}{2 n+3}+\frac{n^{2}}{2 n-1}\right) \\
J_{m n}^{1} & =-\int_{-1}^{1} \frac{2 n+1}{2 n(n+1)} P_{m}^{1}(\eta) P_{n}^{1}(\eta)\left(2 \eta^{2}-1\right) d \eta \\
& =d_{2}(n) \delta_{m, n+2}+d_{0}(n) \delta_{m, n}+d_{-2}(n) \delta_{m, n-2}, \\
d_{2}(n) & =-\frac{2(n+2)(n+3)}{(2 n+3)(2 n+5)}, \quad d_{-2}(n)=-\frac{2(n-2)(n-1)}{(2 n-1)(2 n-3)} \\
d_{0}(n) & =1-\frac{2}{2 n+1}\left(\frac{n(n+2)}{2 n+3}+\frac{(n-1)(n+1)}{2 n-1}\right) \\
J_{m n}^{1} & =0 \text { for } m=0 \text { or } n=0 .
\end{aligned}
$$

\section{References}

[1] Y. H. Pao and C. C. Mow, Scattering of plane compressional wave by a spherical obstacle, J. Appl. Phys. 34, 493 (1963)

[2] C. C. Mow, Transient response of a rigid spherical inclusion in an elastic medium, J. Appl. Mech., Trans. ASME, Ser. E 32, 637 (1965)

[3] C. C. Mow, On the transient motion of a rigid spherical inclusion in an elastic medium and its inverse problem, J. Appl. Mech., Trans. ASME, Ser. E 33, 807 (1966)

[4] L. Knopoff, Scattering of compressional waves by spherical obstacles, Geophysics 24, 30 (1959)

[5] L. Knopoff, Scattering of shear-waves by spherical obstacles, Geophysics 24, 209 (1959)

[6] H. Lamb, Problems relating to the impact of waves on a spherical obstacle in an elastic medium, Proc. London Math. Soc., Ser. 1 32, 120 (1900)

[7] H. Lamb, On a pecularity of the wave-system due to the free vibrations of a nucleus in an extended medium, Proc. London Math. Soc., Ser. 1 32, 208 (1900)

[8] A. E. H. Love, Some Illustrations of modes of decay of vibratory motions, Proc. London Math. Soc., Ser. 2 2, 88 (1904)

[9] P. Chadwick and E. A. Trowbridge, Oscillations of a rigid sphere embedded in an infinite elastic medium, II. Rectilinear oscillations, Proc. Camb. Philos. Soc. 63, 1207 (1967)

[10] A. K. Mal, D. D. Ang and L. Knopoff, Diffraction of elastic waves by a rigid circular disc, Proc. Camb. Philos. Soc. 64, 237 (1968)

[11] S. K. Datta, The diffraction of a plane compressional elastic wave by a rigid circular disc, Q. Appl. Math. 28, 1 (1970)

[12] A. K. Mal, Motion of a rigid disc in an elastic solid, Bull. Seism. Soc. Am. 61, 1717 (1971)

[13] V. A. Erma, An exact solution for the scattering of electromagnetic waves from conductors of arbitrary shape, I. Case of cylindrical symmetry, Phys. Rev. 173, 1243 (1968)

[14] S. K. Datta, Rectilinear oscillations of a rigid inclusion in an infinite elastic medium, Int. J. Engr. Sci. 9, 947 (1971)

[15] S. K. Datta, Torsional oscillations of a rigid inclusion in an infinite elastic medium, in Proc. 3rd Canadian Congr. Appl. Mech. (ed. P. G. Glockner), The University of Calgary, Calgary, Canada, 1971

[16] D. S. Ahluwalia, J. B. Keller and E. Resende, Reflection of elastic waves from cylindrical surfaces, J. Math. and Mech. 19, 93 (1969)

[17] E. G. Lawrence, Diffraction of elastic waves by a rigid inclusion, Quart. J. Mech. Appl. Math. 23, $389(1970)$

[18] S. A. Thau and Y. H. Pao, A perturbation method for boundary value problems in dynamic elasticity, Q. Appl. Math. 25, 243 (1967) 
[19] W. E. Williams, A note on slow vibrations in an elastic medium, Quart. J. Mech. Appl. Math. 19, $413(1966)$

[20] J. B. Keller, R. M. Lewis, and B. D. Seckler, Asymptotic solution of some diffraction problems, Comm. Pure Appl. Math. 9, 207 (1956)

[21] V. D. Kupradze, Dynamic problems of elasticity, in Progress in solid mechanics (ed. I. N. Sneddon and R. Hill), Vol. 3, North-Holland Publishing Co., Amsterdam (1963)

[22] M. Nagase, Diffraction of elastic waves by a spherical surface, J. Phys. Soc. Japan 11, 279 (1956)

[23] F. R. Norwood and J. Miklowitz, Diffraction of transient elastic waves by a spherical cavity, J. Appl. Mech., Trans. ASME, Ser. E 34, 735 (1967)

[24] H. M. Nussenzveig, High-frequency scattering by an impenetrable sphere, Ann. Phys. 34, 23 (1965)

[25] W. M. Ewing, W. S. Jarditzky and F. Press, Elastic waves in layered media, McGraw-Hill, New York (1957)

[26] F. Gilbert, Scattering of impulsive elastic waves by a smooth convex cylinder, J. Acoust. Soc. Amer. 32, 841 (1960) 